\title{
Software Design of Intelligent Detection Platform for Electric Energy Measuring Equipment
}

\author{
CHENG Fu-yong ${ }^{1,2^{*}}$, WANG En ${ }^{1,2}$, ZHAI Shao-lei ${ }^{1,2}$, LI Bo $^{1,2}$, CHEN Zheng ${ }^{1,2}$, and ZHAO Bing-hui ${ }^{3}$ \\ 1. Yunnan Electric Power Research Institute, Kunming 65027, Yunnan Province, China; \\ 2. Key Laboratory of CSG for Electric Power Measurement, Kunming 650217, Yunnan Province, China; \\ 3. Metrology Center of Guangdong Power Grid Corporation, Guangzhou 510000, Guangdong Province, China.
}

\begin{abstract}
This paper aims to effectively detect and estimate the performance of various electric energy measuring equipments. We develop an intelligent detection platform for the electric energy measuring equipment which includes detection software, data storage, intelligent protocol library, virtual network and alarm program. By designing the intelligent protocol library and virtual network, data from different kinds of acquisition terminals can be collected by the proposed intelligent platform. Compared with the conventional electric energy measuring equipment, the intelligent electric energy measuring equipment can greatly save time and improve detection efficiency.
\end{abstract}

\section{Introduction}

With the development of the power industry, various electric energy measuring equipment (EEME) are widely applied in power management systems [1-3]. So far, it is hard to detect different kinds of acquisition terminals for EEME. On the one hand, the product quality of acquisition terminal is quite different and may be poor, leading to difficulty on detection in a short time; on the other, the function of EEME is too unitary, which is unable to systematic detection. Therefore, it is promising to develop an effective and comprehensive detection platform [4-6].

Up to now, the detections of various EEME need different kinds of platforms and only can do some basic performances [7-8]. It is very difficult to compressively estimate the performance and communication of EEME. How to solve this issue has become a focal point in academia. Therefore, we should develop the detection platform for measuring equipment including energy meter, data collator, concentrator, management terminal of distribution transformer and load control. The objective is to synthetic evaluate performance of the above measuring equipment, which improves the efficiency of work.

This study aims to develop an intelligent detection platform for different kinds of EEME or acquisition terminals. The proposed platform includes the detection software, the data storage, the intelligent protocol library, the virtual network and the alarm program. A good communication between hardware and software is achieved. The detection software can independently measure the various electric equipment. The design of the data storage has capability of classifying different kinds of data. The intelligent protocol library is able to realize the closed loop detection and regulation of various measuring equipment and acquisition terminals. The virtual network is to emulate the whole traditional networking in a laboratory and can master power consumption of a building or community. Meanwhile, it detects the meter-reading terminal, the acquisition terminals and the energy meter. Compared with traditional networking, the virtual network can directly read the data of the energy meter and overcomes the disadvantages of long wiring due to the dispersion of various parts. The task of the alarm program reminds the insufficient balance of electric energy meter, monthly flow over limit and detection item. The two salient features of the proposed intelligent detection platform are conceptions of the intelligent protocol library and the virtual network, which is beneficial to improve the detection ability and cut down the cost.

\section{Software design for intelligent detection platform}

Basic on the intelligent detection platform, an intelligent protocol library is introduced. It contains agreements of various EEME and acquisition terminals, and is easy to switch for detecting different kinds of equipment.

In addition, the conception of the virtual network is presented. The virtual network can emulate power consumption of customer in a building or community. It also can detect terminal equipment and electric meter, which is in favour of finding problems without delay and reduce the cost.

\subsection{Framework of software system}

* Corresponding author: futao_phd@163.com 
Fig. 1 depicts the physical frame of intelligent detection platform. It can unify managing the energy equipment and load control equipment. Additionally, it makes the hardware equipment with the same function possess one protocol. Therefore, the communication between the software system and the hardware of this platform is achieved by uniform protocols. This platform independently detects various electric managing equipment, such as load control terminals, concentrator, acquisition terminals and intelligent electric meter of reading data in simulation.

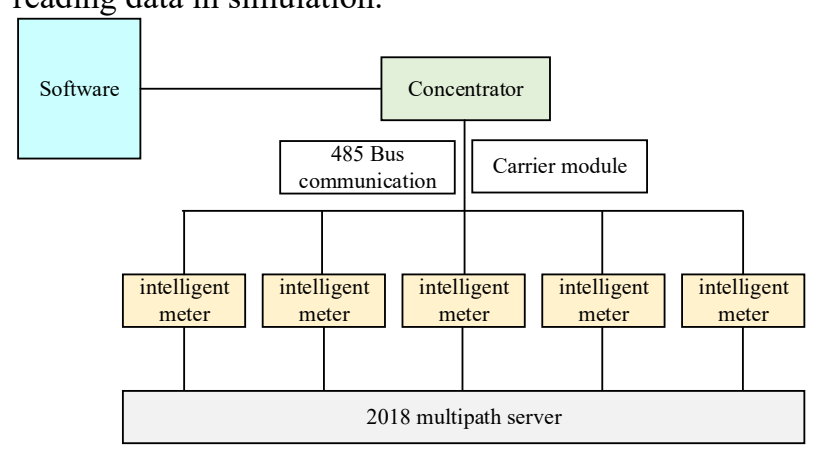

Fig. 1. Physical frame of intelligent detection platform.

Fig. 2 plots logical model chart of the intelligent detection platform. The communication between the computer and the muti-serial port server is achieved by the agreement of TCP/IP. The muti-serial port server connects power supply of CL303 by 485 port. Additionally, the computer can directly link with the terminals by GPRS.

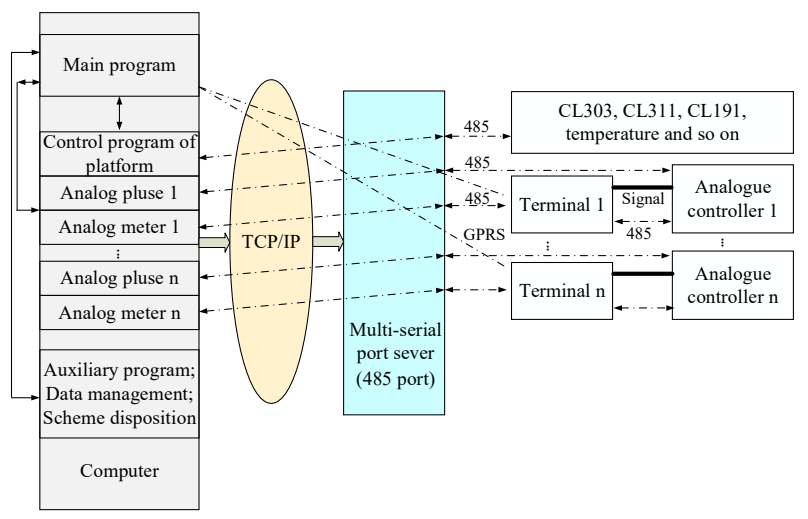

Fig. 2. Logical model chart of the intelligent detection platform.

Fig. 3 shows the software frame of the intelligent detection platform. The communication between the main program, control program, terminal and analog meter is achieved by the agreement of TCP. The mutiserial port server is linked with the simulator of the electric meter and terminal by UDP. It is also connected with terminal by 485 .

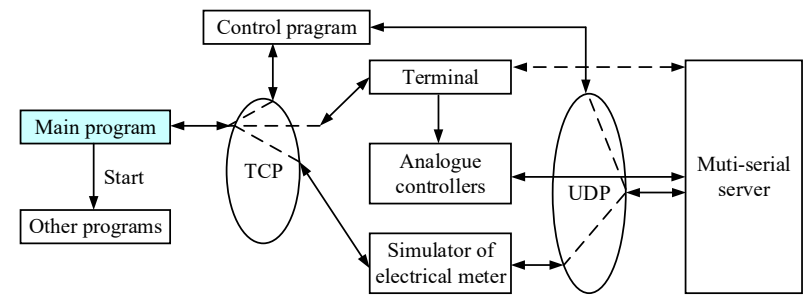

Fig. 3. Software frame of the intelligent detection platform.

The software is composed of standalone program. The main program can exchange with data management and scheme management by data. Other programs is linked with the main program by the agreement of the TCP/IP. The program frame is shown as Fig. 4.

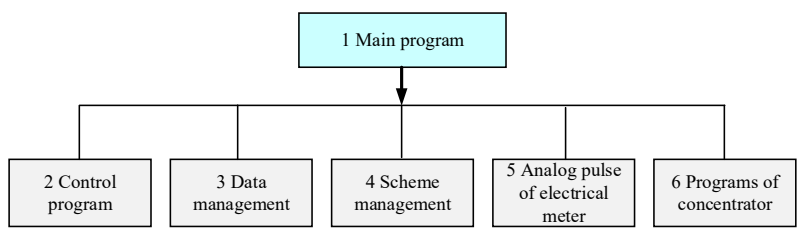

Fig. 4. The program frame of the intelligent detection platform.

The main program is responsible for writing parameters of terminal and detecting control process. Controller for the platform is linked with power source, standard meter and other hardware. The data management can consult various data from equipment, and do backup and recovery operation of data. It also can generate and print report files that meet user requirements. The analog pulse of electric meter transmits the pulse to the terminal and analog switch by the controller. The simulator of the electric meter mainly reads data from electric energy meter of meeting the standard agreement of DL/T645-1997. Meanwhile, it can emulate different kinds of fault of electric meter. The internal framework of main program in the intelligent detection platform is shown as Fig. 5.

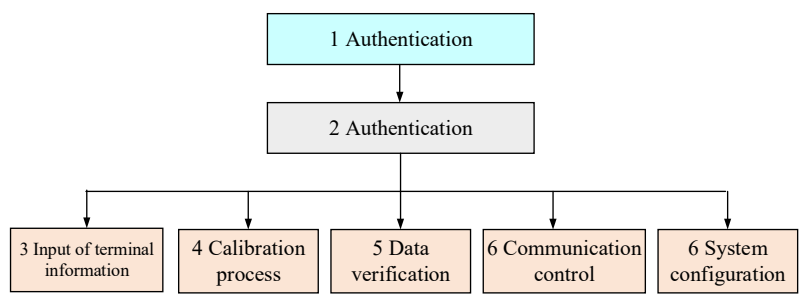

Fig. 5. The internal framework of main program in the intelligent detection platform.

\subsection{Data storage of software system}

Different kinds of data can be classified and stored by the intelligent detection platform. The scheme database of the electric energy meter and the acquisition terminal are built (e.g., ClouConfig.md and Cloufkt_sys.mdb). The calibration for the electric energy meter and the acquisition terminal can depend on the database (e.g., ClouMeterData.mdb and Cloufkt dat.mdb). Fig. 6 and Fig. 7 respectively show the database structure of calibration for the electric energy meter and the acquisition terminal.

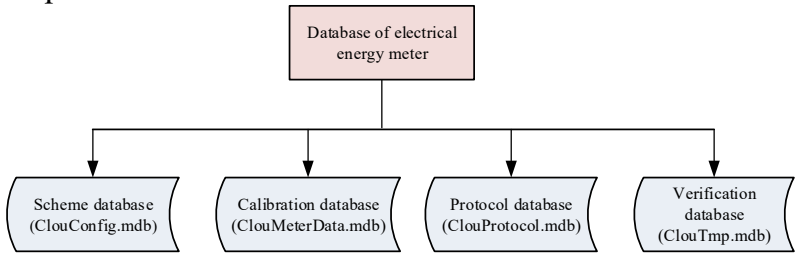


Fig. 6. The database structure of calibration for the electric energy meter.

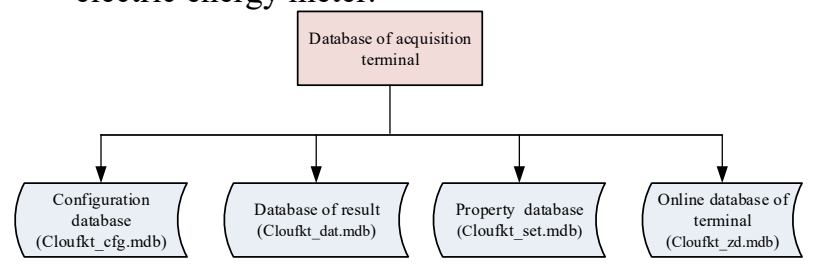

Fig. 7. The database structure of calibration for the acquisition terminal.

The next task is to introduce the testing process in detail for the intelligent detection platform. The specific steps are as follows.

1. Write the parameter. Firstly, various function button including function keys of the interface need to work well. The test condition is list and described, such as auto number, special replacement of terminal. In testing process, we should correctly choose the way of measurement (i.e., current or voltage), and software program should be corresponding to counterpart of testing program. Then, the fault tolerance test is implemented for possibly wrong operation. For example, the voltage $1.5 \mathrm{v}$ is wrong written $6 \mathrm{v}$. In addition, the data integrity should be checked by the fault tolerance.

2. Set system parameter. Firstly, the set parameters should be ensure correctness and can be saved. Various inputs of possible illegal parameter are emulated and validated the automatic functions of the identification and adjustment. Then, the parameter of electric meter is set. It is observed that the reading of analog electric meter changes with the parameter.

3. Manage the data. Firstly, we check the different kinds of function buttons including the interface. The detailed explanation of testing condition is presented and printed out. Then, testers verify the appearance of report on data integrity, and make sure that the report is intuitive, neat and convenient.

4. Manage the scheme. Firstly, the test items and subitems of scheme on addition, deletion and duplication should be validated. Then, the verification about the default values of scheme need to carry out. Furthermore, the only sub-scheme will be deleted if the scheme is deleted.

5. Test the function. Firstly, single test function should be operated and cannot produce unexpected error. The phenomenon is able to consist with the result descried by the program. Then, automatic test of all functions is working properly and is guaranteed that it will not violate the program.

6. Conduct the comprehensive test. Firstly, the integrity of the whole program is tested. Whether the combination between the main program and the subprogram is feasible. Also, the button function is checked and the fault tolerance test is conducted. Theoretically, the input errors of the current and the voltage are not allowed and other operations of software can identify the above error and start protection measures. In the test process, tester do not follow normal operation, deliberately. This operation can detect the fault tolerance.
7. Emulate the pulse of the electric meter. Firstly, it should make sure that the analog meter is only a subsidiary program when the main program is running. Except for querying the value of the electric meter, the analog meter cannot do other works. Then, the analog meter of the pulse is an independent program to finish testing work if the main program is closed.

\subsection{Intelligent protocol library}

Base on the intelligent detection platform, the intelligent protocol library can realize the closed loop detection for different kinds of the electric energy measuring equipment and acquisition terminals. Fig. 8 shows the design of the intelligent protocol library for the electric energy measuring equipment.

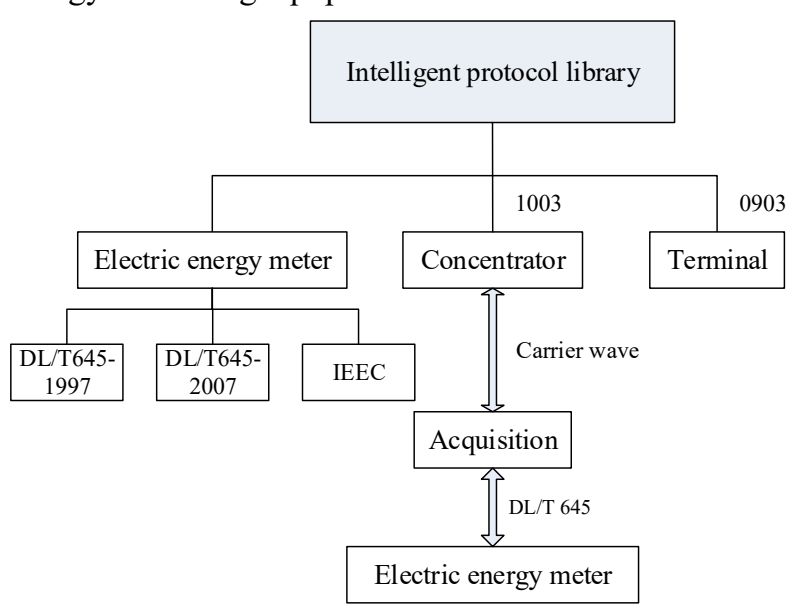

Fig. 8. The intelligent protocol library for the electric energy measuring equipment.

In general, electric energy meter of muti-functions mainly adopts the agreement of DL/T645-1997, DL/T645-2007 and IEEC. However, the detection software of the developed intelligent electric meter integrates all agreements. Furthermore, meter reading terminal follows the standard (Q/GDW 376.1). The communication between the concentrator and the acquisition is achieved by the carrier wave. The interactions between the acquisition and the electric energy meter should meet the agreement of DL/T645. Therefore, the intelligent detection platform can be widely used for detecting electric energy measuring equipment by combing the intelligent protocol library.

\subsection{Virtual network}

Generally, traditional network is to collect the data of consumer's electricity meter by using the terminal in the station from the carrier wave, acquisition and RS485. Then, the host computer can analyse the data from the terminal. However, it is difficult to directly read data of the electric meter, owing to all parts of the traditional network are relatively scattered and the wiring is relatively long.

Compared with the traditional network, the virtual network can emulate the whole traditional network in one laboratory. It integrates meter reading terminal, 
collector and electric energy meter on one bench. By combining lines RS485, the data from the acquisition terminal and consumer's electricity meter is collected. Therefore, the virtual network not only emulates the scenario of power consumption in a building or community, but also detects meter reading terminal, concentrator and electric energy meter. Fig 9 and Fig. 10 respectively plot structure chart of the traditional network and the virtual network.

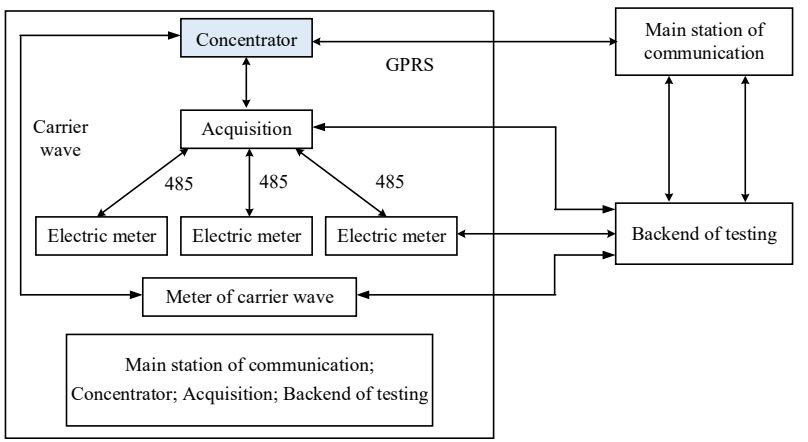

Fig. 9. The structure chart of the traditional network.

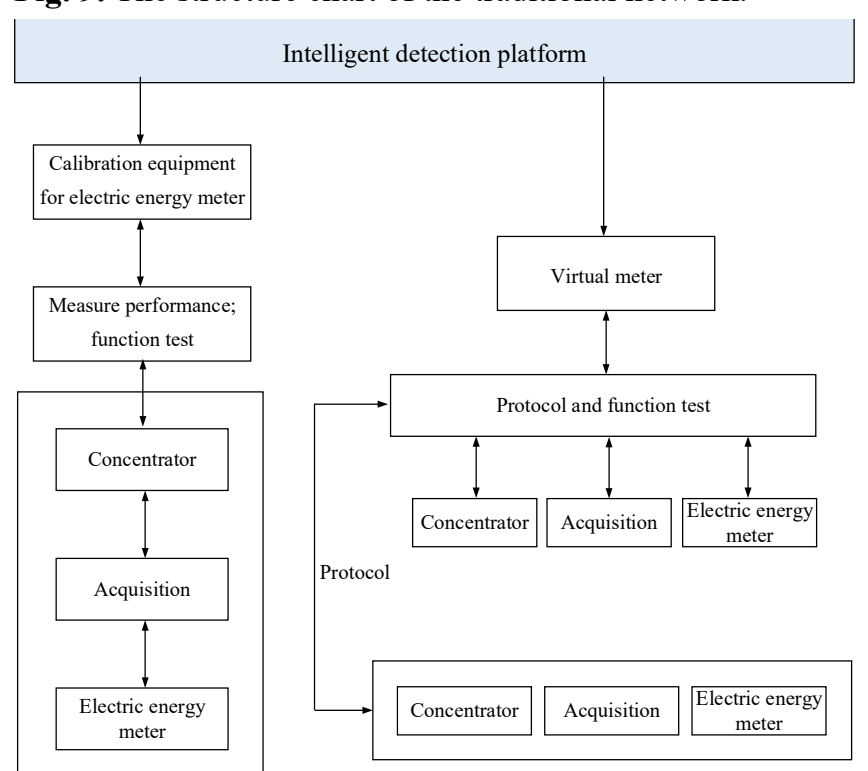

Fig. 10. The structure chart of the virtual network.

\subsection{Alarm program}

Compared with the old verification regulations, the new verification regulations has been added some detection items, such as, alarm for insufficient balance of electric energy meter, alarm for monthly flow over limitation, alarm for the open of the power meter box, terminal power down under no battery, parameter comparison with and without power down, voltage unbalance alarm, standby sleep test, power performance test and alarm of reading failure.

The following is an example of the alarm when the remaining amount of the electricity meter is insufficient. The implementation of the intelligent detection platform is described in detail.

Through the analog technology of electric energy meter, the alarm signals can be addressed. The whole test process adopts the positive and negative verification method. First, the remaining amount of the analog meter is set to a large random number. The random number should be greater than the alarm threshold value, which is set to 500 in the process. If the function of the terminal is normal, the alarm signal is empty by controlling the remaining amount of the electricity meter by the tested terminal. This step verifies whether the terminal under test will generate false alarm signal when the meter under test is normal. Second, the remaining amount of the analog meter is modified in the testing process. The remaining amount of the analog meter is set to 1 , which is smaller than the alarm threshold. Meanwhile, data of analog meter at control terminal is read. If the event is recorded normally, it is determined that the alarm function is effective in the terminal reading for the remaining amount of the electric energy meter. Otherwise, it is considered to be unqualified. Fig. 11 shows the testing flow chart of the alarm for the remaining amount of electric energy meter.

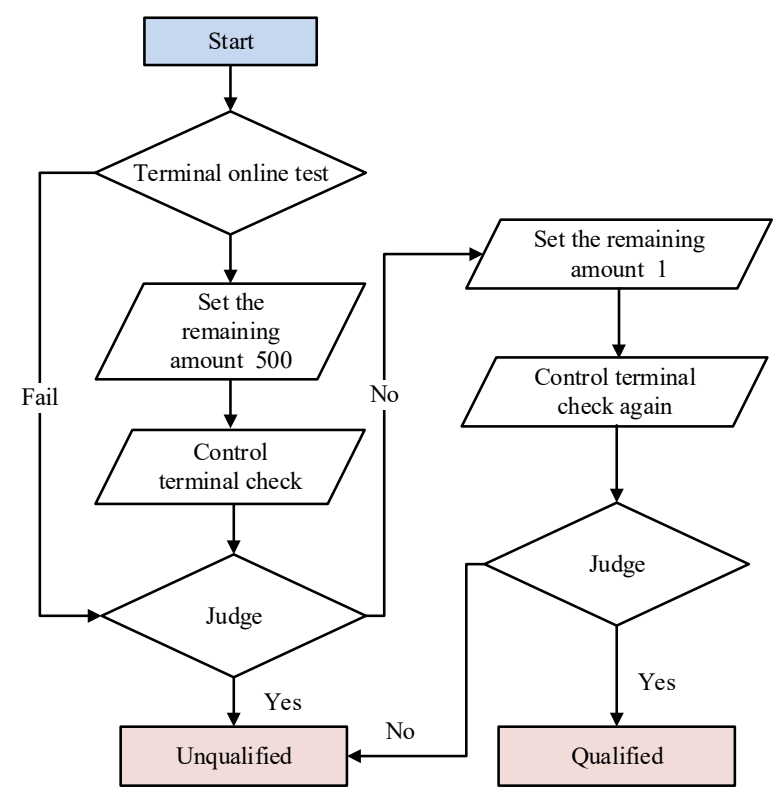

Fig. 11. The testing flow chart of the alarm for the remaining amount of electric energy meter.

\section{Conclusion}

This paper mainly introduces the intelligent detection platform for EEME, including detection software, data storage, intelligent protocol library, virtual network and alarm program. The two salient features of the proposed platform are that the conceptions of the intelligent protocol library and the virtual network are presented. The intelligent protocol library can realize the closed loop detection for different kinds of the EEMEs and acquisition terminals, which includes a rather wide range of practical needs. On the other hand, the virtual network not only emulates the scenario of power consumption in a building or community, but also detects meter reading terminal, concentrator and electric energy meter. This characteristic is employed to distinguish between the traditional network and the virtual network. In the testing 
process, it can be found that compared with the traditional detection platform, the intelligent detection platform can greatly save time and improve detection efficiency.

\section{References}

1. Y. Gong, H. Y. Liu, X. Yin, X. G. Li, Y. H. Liang, J. X. Man, R. X. Wen. Research on verification platform of electric energy metering equipment operated in typical environment. IOP Conference Series: Materials Science and Engineering. 2018, 439(5): 052020.

2. Y. He, Z. Wang, Y. Wang, C. Li, C. Shi, L. Zou. Research on design of a fully compatible electric energy meter and power information acquisition equipment tray. IEEE Sustainable Power and Energy Conference (iSPEC). 2019, pp. 2605-2608.

3. H. C. Sun, L. Y. Zhang, W. F. Zhang, S. J. Wang. Research on the energy-controlling system for the terminal electric equipment in office building. Procedia Engineering. 2017, 205: 281-287.

4. L. Yang, D. Zhang, G. Lin, Q. Song, Q. Meng, Q. Pan, C. Liu. Research on remote calibration and online monitoring system of electric energy metering device. Journal of Physics: Conference Series. 2019, 1303(1): 012119.

5. A. Berouine, F. Lachhab, Y. N. Bakhouya, R. Ouladsine. A smart metering platform using big data and IoT technologies. 2017 3rd IEEE International Conference of Cloud Computing Technologies and Applications, 2017, pp. 1-6.

6. S. Huai, L. Yan, Z. Ang, Q. Song, Z. Wu. Research on technical architecture and application of big data cloud platform for electric power measurement. Journal of Physics: Conference Series. 2019, 1213(4): 042040.

7. S. L. Gong, Y. L. Wang, M. Y. Zhang. Design of intelligent power consumption optimization and visualization management platform for large buildings based on internet of things. MATEC Web of Conferences. 2017, 139: 00119.

8. Li, Peng, et al. Design of large-scale platform detection system based on distributed wireless communication technology. 2018 3rd Joint International Information Technology, Mechanical and Electronic Engineering Conference (JIMEC). 2018. 